\title{
Réhabilitation sur implants après reconstruction mandibulaire par greffon de fibula microanastomose : une série de 10 cas
}

\author{
Implant rehabilitation after reconstructed mandible by microvascular \\ fibula graft: a series of 10 cases
}

\author{
ANNE-GAËLLE BODARD*, RENÉ GOURMET*
}

\begin{abstract}
RÉSUMÉ
Objectifs : L'utilisation du greffon de fibula microanastomosé a transformé les possibilités de réhabilitation prothétique après mandibulectomie interruptrice. Son volume, sa faible résorption et ses taux de succès permettent d'envisager la mise en place d'implants dentaires supportant une prothèse adjointe ou conjointe. Méthode : Cette série comporte 10 patients ayant bénéficié d'une reconstruction mandibulaire par lambeau de fibula microanastomosé, puis de la mise en place d'implants, et enfin d'une réhabilitation prothétique.

Résultats : Sur les 10 patients, 8 ont été traités pour un carcinome épidermoïde des voies aéro-digestives supérieures, 1 pour une histiocytose $X$ et 1 pour un granulome à cellules géantes. 29 implants ont été mis en place ; 1 a été perdu. 2 patients ont été réhabilités par prothèse conjointe, les 8 autres par prothèse adjointe. Le recul clinique varie de 9 à 61 mois.

Discussion : Le délai entre la greffe et la mise en place des implants est de 11 à 21 mois; selon les auteurs le délai recommandé varie de 4 à 24 mois.

L'épaisseur des tissus mous oblige à choisir des piliers très longs ; une prolifération tissulaire autour des piliers implantaires peut être observée.

Conclusion : Cette série constitue une approche positive des possibilités de réhabilitation supra-implantaire sur lambeau de fibula microanastomosé, permettant le rétablissement des fonctions buccales et l'esthétique faciale, constituant une étape essentielle pour améliorer la qualité de vie des patients. (Med Buccale Chir Buccale 2005; 11: 215-221).
\end{abstract}

mots clés : Implants dentaires, reconstruction mandibulaire, lambeau de fibula microanastomosé,réhabilitation prothétique

\section{SUMMARY}

Objective: The use of microvascular fibula graft has transformed prosthetic rehabilitation after interruptive mandibular surgery. Its volume, its poor resorption and its success allow the use of dental implants supporting a fixed or movable prosthesis.

Methods: This series is composed of 10 clinical patients who have undergone mandibular reconstruction with a free fibula flap followed by dental implants and prosthetic rehabilitation.

Results: 8 patients were treated for an oral cancer, 1 for a histiocytose $X$ and 1 for a giant cell tumor. 29 implants were placed; 1 was lost. 2 fixed prostheses were realized and 8 movable prosthesis. Clinical follow up is from 9 to 61 months. Discussion: Delay between graft and implantation is from 11 to 21 months; authors recommend a delay from 4 to 24 months. Thickness of soft tissues leads to choose long abutments; a tissular proliferation might be observed around the abutments.

Conclusion: This series is a positive approach of implant-supported prosthesis on a microvascular fibula graft, restoring oral functions and orofacial aesthetics as an essential step in increasing the patient's quality of life. (Med Buccale Chir Buccale 2005; 11 : 215-221).

key words : Dental implants, mandibular reconstruction, microvascular fibula graft, prosthetic rehabilitation

*Unité d'Odontologie -Service de Chirurgie, Centre Léon Bérard, Lyon

Demande de tirés à part :

Dr Anne-Gaëlle Bodard, Unité d'Odontologie, Service de Chirurgie, Centre Léon Bérard, 28 rue Laennec, 69373 Lyon Cedex 08 , e-mail : bodard@lyon.fnclcc.fr 
médecine

buccale

chirurgie

buccale

VOL. $11, \mathrm{~N}^{\circ} 4$ 2005

page 216
La mandibulectomie ou la pelvimandibulectomie interruptrice a de multiples répercussions sur les fonctions buccales et l'esthétique faciale. Dans un cas l'hémimandibule résiduelle est attirée côté réséqué, dans l'autre cas les deux fragments mandibulaires résiduels tendent à se rapprocher. La latérodéviation a pour conséquence une perte de l'articulé dentaire, une diminution du coefficient masticatoire et un effacement du gonion et du menton cutané [1]. Les objectifs de la chirurgie reconstructrice sont multiples:

- rétablir la continuité mandibulaire,

- restituer un volume osseux donc une forme anatomique adéquate,

- prévenir la résorption ultérieure de la greffe,

- supprimer les défauts des tissus mous,

- restituer un contour facial harmonieux et symétrique,

- donner une meilleure assise à la prothèse ultérieure,

- permettre une normalisation des fonctions buccales.

La chirurgie mandibulaire reconstructrice permet de restaurer l'arc anatomique. De plus, elle fournit un support prothétique satisfaisant ; le recours à des techniques implantaires permet d'ancrer la prothèse, d'éviter un appui muqueux trop important et donc de rétablir la fonction masticatoire et l'esthétique. Le greffon microanastomosé de fibula a été utilisé pour reconstituer la mandibule pour la première fois par Hidalgo en 1989 [2]. La fibula est un os cortical, triangulaire dont la section, variant de 10 à $13 \mathrm{~mm}$, autorise la mise en place d'implants [3]. Cette étude se propose d'évaluer les résultats de la mise en place d'implant sur un greffon de fibula, notamment après radiothérapie cervico-faciale.

\section{MATÉRIEL ET MÉTHODE}

Cette série comporte 10 patients, traités par mandibulectomie ou pelvimandibulectomie interruptrice. La chirurgie d'exérèse a été suivie par une reconstruction par un greffon de fibula microanastomosé, puis par la mise en place d'implants dentaires et d'une réhabilitation prothétique fixe ou amovible. Parmi ces 10 patients, 8 ont été traités pour un carcinome épidermoïde des voies aéro- digestives supérieures, 6 d'entre eux ont eu une radiothérapie avant et 2 après la greffe (patients 4 et 5) ; 1 patient a été traité pour un granulome à cellules géantes et un pour une histiocytose $X$. L'âge des patients est compris entre 16 et 61 ans. La série se compose de 9 hommes et 1 femme.

Le protocole clinique est le suivant :

- examen clinique : on évalue l'ouverture buccale, les fonctions linguales, l'existence ou non d'un vestibule, sa profondeur, la mobilité labiale, l'épaisseur de la palette musculo-cutanée...

- examen radiologique : orthopantomogramme et examen tomodensitométrique.

Les implants sont mis en place sous anesthésie générale afin d'optimiser les conditions d'hygiène et préserver la vascularisation locale, éventuellement altérée par la radiothérapie. Les systèmes utilisés sont des systèmes enfouis, en deux temps chirurgicaux. Le forage est effectué sous irrigation abondante et la mise en place des implants, manuelle ou mécanique, à vitesse lente. Des sutures hermétiques sont réalisées et une antibiothérapie (débutée pendant le geste opératoire en V) est poursuivie pendant 8 à 10 jours, jusqu'à l'obtention de la cicatrisation muqueuse. Un contrôle clinique et radiographique est effectué à J8, puis à J30 et J90. A 6 mois, le deuxième temps opératoire est effectué sous anesthésie locale et la réalisation prothétique commence 15 jours plus tard. La chirurgie implantaire et les étapes prothétiques sont réalisées par la même équipe.

\section{RÉSULTATS (Tab. I)}

L'âge moyen dans notre série est de 44 ans. Le plus jeune patient a 16 ans et le plus âgé 61 ans. 29 implants ont été mis en place : 11 implants de type Ciny ${ }^{\circledR}$ (Serf) et 18 de type MKIII ${ }^{\circledR}$ (NobelBiocare). Le délai entre la greffe et l'implantation est compris entre 8 et 33 mois, le délai moyen étant de 20,4 mois.

Pour les patients ayant bénéficié d'une radiothérapie cervico-faciale, les doses sont comprises entre 56 et 65 Grays, avec une moyenne de 59,6 Grays. Le délai entre la fin de la radiothérapie et l'implantation est compris entre 12 et 48 mois, avec un délai moyen de 29,3 mois. 
Tableau 1 : Résultats : présentation des cas cliniques Results: presentation of clinical cases

\begin{tabular}{|c|c|c|c|c|c|c|c|c|c|}
\hline $\begin{array}{c}\mathrm{N}^{\circ} \\
\text { patient }\end{array}$ & $\begin{array}{l}\text { Sexe } \\
\text { (M/F) }\end{array}$ & $\begin{array}{l}\text { Age } \\
\text { (ans) }\end{array}$ & $\begin{array}{l}\text { Délai greffe/ } \\
\text { implants }\end{array}$ & $\begin{array}{l}\text { Délai RT/ } \\
\text { implants }\end{array}$ & $\begin{array}{c}\text { Dose RT } \\
\text { (Grays) }\end{array}$ & $\begin{array}{l}\text { Nombre } \\
\text { implants }\end{array}$ & $\begin{array}{l}\text { Diamètre } \mathrm{x} \\
\text { longueur } \\
\text { implants }\end{array}$ & $\begin{array}{c}\text { Type } \\
\text { implant } \\
\text { (Serf/Nobel) }\end{array}$ & $\begin{array}{c}\text { Prothèse } \\
\text { adjointe (PA)/ } \\
\text { conjointe (PC) }\end{array}$ \\
\hline 1 & M & 50 & 24 & 12 & 56 & 2 & $3 \times 10$ & S & PA \\
\hline 2 & M & 38 & 22 & 24 & 60 & 3 & $3 \times 10$ & S & PA \\
\hline 3 & M & 61 & 24 & 26 & 65 & 2 & $3 \times 10$ & S & PC \\
\hline 4 & M & 41 & 33 & 30 & 60 & 2 & $3,75 \times 10$ & N & PA \\
\hline 5 & $M$ & 48 & 17 & 48 & 60 & 2 & $3,75 \times 10$ & N & PA \\
\hline 6 & M & 44 & 11 & 24 & 60 & 2 & $3 \times 10$ & S & PA \\
\hline 7 & M & 46 & 24 & 43 & 56 & 4 & $3,75 \times 10$ & N & PA \\
\hline 8 & $M$ & 41 & 8 & 29 & 60 & 2 & $3 \times 10$ & S & PA \\
\hline 9 & M & 16 & 11 & - & - & 8 & $3,75 \times 10$ & N & PC \\
\hline 10 & $\mathrm{~F}$ & 56 & 20 & - & - & 2 & $3 \times 12$ & S & PA \\
\hline
\end{tabular}

6 patients ont été irradiés avant la greffe, 2 après la greffe (mais 12 et 24 mois avant la mise en place des implants).

Sur les 29 implants, 1 ne s'est pas ostéo-intégré (patient 9) : il a été déposé lors du deuxième temps chirurgical. Les implants utilisés ont les dimensions suivantes : $3 \times 10 \mathrm{~mm}\left(\right.$ Ciny $\left.{ }^{\circledR}\right)$ et $3,75 \times 10 \mathrm{~mm}\left(\mathrm{MKIII}{ }^{\circledR}\right)$. Le nombre d'implants par patient varie de 2 à 8, la moyenne étant de 2,9 par patient. 2 patients ont été réhabilités par prothèse conjointe, 6 par prothèse adjointe partielle stabilisée et 2 par prothèse complète stabilisée. Le recul clinique est compris entre 9 et 61 mois, la moyenne étant de 33 mois.

\section{DISCUSSION}

Cette série se compose de patients âgés de 16 à 61 ans, avec une moyenne d'âge de 44 ans. II est intéressant de noter que les patients traités pour un carcinome épidermoïde sont exclusivement des hommes, avec un âge moyen de 46 ans. Le greffon microanastomosé de fibula présente de nombreux avantages :

- prélèvement simple,

- longueur osseuse disponible importante,

- volume et épaisseur adaptés à la reconstruction mandibulaire,

- restauration des fonctions satisfaisante,
- possibilité d'effectuer la greffe dans le même temps que l'exérèse tumorale,

- faible morbidité sur les sites donneurs et receveurs,

- possibilité de mettre en place des implants dentaires,

- possibilité de modelage par ostéotomies du greffon,

- intervention chirurgicale avec deux équipes sans changement de position opératoire,

- faible résorption osseuse.

Les inconvénients d'un tel greffon sont:

- mobilité et finesse de la palette cutanée,

- épaisseur importante du tissu sous-cutané,

- absence de vestibule et de sillon glosso-pelvien,

- séquelles esthétiques surtout s'il y a eu une greffe de peau pour fermer la prise de la palette cutanée,

- utilisation uniquement de petits implants, avec un ratio couronne-racine défavorable.

Dans une étude sur pièce anatomique, Niimi ${ }^{[4]} \mathrm{a}$ montré que :

- les os les plus adaptés en terme de dimensions et volume sont la crête iliaque et la fibula,

- la force d'arrachement d'un implant est plus importante dans la fibula que dans la scapula et la crête iliaque,

- la dureté globale de l'os est supérieure pour la fibula, puis par ordre décroissant la crête iliaque et la scapula. médecine

buccale

chirurgie

buccale

VOL. $11, N^{\circ} 4$ 2005

page 217 
Pour Sclaroff [5], la fibula est l'os le mieux adapté pour la reconstruction mandibulaire, tandis que l'os iliaque est optimal pour la reconstruction maxillaire. Le prélèvement du pédicule nourricier et la microanastomose permettent la conservation de la viabilité tissulaire, une meilleur résistance aux infections et aux traumatismes, et une cicatrisation osseuse comparable à celle d'une fracture.

Le greffon microanastomosé constitue un site correct pour placer des implants. II possède sa propre vascularisation et présente une résorption osseuse similaire à celle d'une mandibule normale, soit $0,2 \mathrm{~mm}$ pour 2 ans [6]. Par ailleurs, Serra [7] a montré que le greffon de fibula microanastomosé tolère bien la radiothérapie ou la chimiothérapie. Si la radiothérapie a lieu avant la greffe, la vascularisation et les cellules osseuses du greffon ne sont pas altérées.

médecine buccale chirurgie buccale

VOL. $11, \mathrm{~N}^{\circ} 4$ 2005

page 218
La hauteur d'os disponible est comprise entre 10 et $13 \mathrm{~mm}$, ce qui oblige à placer des implants relativement courts. Néanmoins certains auteurs n'hésitent pas à exploiter toute la hauteur du greffon en plaçant des implants bicorticaux [8]. Les résultats des séries cliniques publiées donnent d'excellents pourcentages tant au niveau clinique qu'histologique [9,10] : par exemple, 97,5\% des résultats satisfaisants pour Sclaroff [5]. Néanmoins les séries cliniques sont petites et ne permettent pas une analyse objective. En territoire irradié, la capacité intrinsèque de cicatrisation et de réparation d'un tel greffon permet de compenser la vascularisation parfois altérée des territoires environnants et évite les défauts d'intégration du gref- fon par manque de néovascularisation (par défaut de " creeping " vasculaire) comme dans les greffes libres [11]. De même, la résorption du greffon est plus faible avec les lambeaux microanastomosés. Par contre, un des inconvénients du lambeau de fibula est qu'il laisse, chez le patient partiellement denté, un décalage vertical important du niveau crestal entre les segments reconstruits et la mandibule résiduelle [12]. Pour Chiapasco [12] les indications d'un greffon vascularisé par rapport à une greffe libre sont les suivants :

- antécédents d'irradiation,

- perte de substance de plus de $9 \mathrm{~cm}$ de long,

- qualité et quantité insuffisantes des tissus receveurs,

- nécessité de greffer aussi des tissus mous.

Certains auteurs [13] (Tab. 2) ont comparé la quantité d'os disponible et la qualité osseuse en fonction des différents sites de prélèvement ; la fibula et la crête iliaque semblent offrir les meilleurs résultats tant au niveau qualitatif (os très cortical) que quantitatif. L'épaisseur plus importante de la corticale fibulaire et la faible quantité d'os spongieux en font un os moins résorbable que l'os iliaque.

Le délai entre la mise en place des implants et la chirurgie est soumis à controverse ; pour certains, un délai minimal d'une année est requis $[14,15]$ afin d'être sûr de la bonne intégration du greffon. Pour d'autres, la mise en place des implants peut se faire dans le même temps que la greffe, d'autant plus qu'il s'agit d'un lambeau vascularisé, donc a priori non soumis aux risques de nécrose ou de

Tableau 2 : Mesures des lambeaux osseux d'après Matsuura [13]

Measurements of osseous flaps by Matsuura [13]

\begin{tabular}{|c|c|c|c|c|}
\hline & Fibula & Crête iliaque & Scapula & Radius \\
\hline Epaisseur & 10,4 à 11,5 mm & 11,1 à 15 mm & 7,5 à 9 mm & 6,1 à 6,3 mm \\
\hline Hauteur & 14 à 16 mm & $26,7 \mathrm{~mm}$ & 13,2 à 19,5 mm & 11,7 à $12,2 \mathrm{~mm}$ \\
\hline Epaisseur corticale & 3,5 à 4,1 mm & $2,55 \mathrm{~mm}$ & 0,8 à 2,8 mm & 2,8 à 3,3 mm \\
\hline Index & $85 \% \mathrm{H}$ & $>90 \%$ & $60 \%$ & $41 \% \mathrm{H}$ \\
\hline d'implantabilité* & $44 \% \mathrm{~F}$ & & & $0 \% \mathrm{~F}$ \\
\hline
\end{tabular}

* L'index d'implantabilité est une estimation de la possibilité de mettre en place des implants et prend en compte notamment le volume et la densité osseuse. 
rejet. Dans la série rapportée, tous les implants ont été mis en place dans un second temps, et non dans le même temps que la greffe osseuse, pour plusieurs raisons. Tout d'abord, ceci permet d'être certain de la bonne intégration du greffon à la mandibule; le positionnement des implants peut être réalisé avec précision et en fonction des impératifs prothétiques, tout en diminuant les aléas chirurgicaux; enfin, dans cette étude, la greffe et l'implantation sont effectuées par des équipes différentes.

La taille des implants est dictée par les dimensions de la greffe. Les auteurs s'accordent sur le fait que les implants mis en place dans les greffons de fibula ne peuvent pas excéder $13 \mathrm{~mm}$ de longueur [16]. Sclaroff [5] observe que les longueurs les plus utilisées sont comprises entre 10 et $13 \mathrm{~mm}$. Les résultats obtenus sont indépendants du système implantaire utilisé et ils sont identiques à ceux retrouvés dans la littérature ; il en est de même pour les dimensions des implants. Pour les patients ayant bénéficié de radiothérapie externe, le délai entre la fin de la radiothérapie et la mise en place des implants est en moyenne de 29 mois. Les auteurs s'accordent sur le fait qu'un délai minimum de 9 mois entre la fin de la radiothérapie et la mise en place des implants est nécessaire [16], afin de permettre au potentiel réparateur des tissus de recouvrer un niveau acceptable. En effet, l'étude de Schweiger [17] sur la souris a montré que la vascularisation osseuse redevenait de meilleure qualité à partir du $9^{\mathrm{e}}$ mois après la fin de la radiothérapie. D'autres [2] préfèrent attendre 1 an après la fin de la radiothérapie. De nombreuses études ont été réalisées sur de la mise en place d'implants en territoire irradié $[18,19,20,21,22,23]$ mais les données sont difficilement interprétables car on ne connaît pas toujours avec précision la dose et le champ d'irradiation, le site d'implantation et le délai après la fin de la radiothérapie. Plusieurs études chez le chien ont été conduites : Aisikainen [18] a montré qu'une dose de 60 Gy entraîne la perte de 50 \% des implants à 5 mois et demi ; par contre une dose de 40 Gy hyperfractionnée stimulerait le remodelage osseux. D'un point de vue histologique, il a été montré [24, 25] que l'ostéointégration est obtenue après une irradiation à dose thérapeutique, mais l'équilibre entre l'apposition et la résorption osseuses n'est retrouvé que 8 mois après la fin de la radiothérapie. L'existence de zones fortement remaniées au sein du tissu osseux irradié a été mise en évidence dans cette même étude. Le délai entre la fin de la radiothérapie et la mise en place d'implants est de 6 à 18 mois pour Granström [26] ; pour Taylor et Worthington [27], il est de 2 ans. Il a montré que l'irradiation entraîne une dépression initiale de la régénération osseuse. Une dose de 5 Gy réduit de plus de $20 \%$ la régénération osseuse, mais celle-ci réaugmente avec le temps : ainsi, elle redevient normale au bout de 15 semaines après avoir reçu 15 Gy. Elle est multipliée par 2,5 un an après la fin de l'irradiation. Brogniez [20] a montré que l'irradiation à dose thérapeutique n'empêche pas une réponse osseuse adaptée à la chirurgie implantaire. L'augumentation du délai donne plus de fiabilité mais peut démotiver le patient.

Dans la série présentée, pour un des patients (patient 7), la radiothérapie a été complétée par une curiethérapie sur le site tumoral initial (plancher buccal antérieur). Tous ont reçu des doses supérieures ou égales à 56 Gy ; la dose moyenne est de 59,5 Grays. Deux patients ont été traités par radiothérapie après la mise en place du greffon de fibula et aucune différence n'a été observée du point de vue clinique. Ceci corrobore les résultats de la littérature.

L'utilisation de l'oxygénothérapie hyperbare est controversée. Pour certains auteurs [26], elle améliore un peu la cicatrisation osseuse mais elle est surtout favorable à la cicatrisation des tissus mous. Cet adjuvant thérapeutique n'a pas utilisé dans l'étude, son efficacité réelle est contestée et elle engendre un surcoût (temporel et financier) et présente de nombreuses contre-indications [28].

Le protocole implantaire proposé par Sclaroff [5] comporte les phases les suivantes:

- exérèse chirurgicale et greffe avec mise en place des implants,

- période de cicatrisation (4 à 6 semaines),

- radiothérapie (généralement 60 Gy),

- période de cicatrisation (6 mois),

- mise en place des piliers, médecine

buccale

chirurgie

buccale

VOL. $11, \mathrm{~N}^{\circ} 4$ 2005

page 219 
- période de cicatrisation (4 semaines), - phase prothétique.

Ce protocole diffère peu de celui utilisé dans cette série. Quelques cas cliniques ont été récemment traités avec une implantation dans le même temps que la greffe, donc avant la radiothérapie. Les résultats préliminaires sont encourageants.

Dans cette série, un implant a été perdu : le taux de succès est donc de $96,5 \%$. Ce taux est évalué après la mise en charge des implants et avec un recul moyen après implantation de 33 mois, soit presque 3 ans. Sachant que la mise en charge finale se fait environ 6 mois après la phase chirurgicale, le recul par rapport à la mise en fonction est de 27 mois environ, avec des extrêmes de 3 à 55 mois. Le taux de succès retrouvé dans la littérature est de $80 \%$ [23] à $99 \%$ [21] ; ce taux est principalement influencé par l'association ou non de la chirurgie avec la radiothérapie (et les médecine chirurgie buccale

VOL. $11, \mathrm{~N}^{\circ} 4$ 2005

page 220 buccale doses de celle-ci). Le taux de succès de cette série est à nuancer, dans la mesure où elle ne présente qu'un petit nombre de cas et d'implants et que le recul pour certains patients est encore faible (2 patients bénéficient d'un recul inférieur à 1 an).

Pour la réhabilitation prothétique, les piliers transgingivaux doivent être très hauts, entre 7 et $10 \mathrm{~mm}$ pour Sclaroff [5]. Ceci est dû à l'épaisseur du tissu sous-cutané apporté par le greffon. Sclaroff conseille également de ne pas réaliser des prothèses avec appui muqueux mais uniquement des prothèses implanto-supportées. En

\section{RÉFÉRENCES}

1 - ATKINSON HF, SHEPERD FW. Masticatory movements of patients after major oral surgery J Prosth Dent 1969 ; $21: 86-91$.

2 - Buchilnder D, URKen M, Vickery C, Weinberg $\mathrm{H}$, SHEINER A, BILleR H. Functional mandibular reconstruction of patients with oral cancer. Oral Surg Oral Med Oral Pathol 1989 ; 68 : 499-504.

3 - GuerRa MFM, Fias LN, Rodriguez FJ, CAmpo FJR, GonZALEZ FJD. Vascularized free fibular flap for mandibular reconstruction: a report of 26 cases. J. Oral Maxillofac Surg $2001 ; 59$ : 140-4.

4 - Niımı A, OZEkI K, Ueda M, Nakayama B. A comparative study of removal torque of endosseous implants in the effet, le risque d'ulcération traumatique de la muqueuse, très fine sur ces lambeaux, doit être évité sous peine de créer une porte d'entrée bactérienne.

Dans notre série, 2 patients ont bénéficié d'une réhabilitation par prothèse conjointe (bridge), les 8 autres ont été réhabilités par prothèse adjointe stabilisée sur implants ( 2 seulement par prothèse adjointe totale, cas 6 et 7). La stabilisation des prothèses a été obtenue par des barres type Ackers ou fraisées; des cavaliers métalliques ou en Téflon ${ }^{\circledR}$ assurent la rétention dans l'intrados prothétique. 2 implants ont été mis en place pour la stabilisation des prothèses adjointes partielles ; un appui dentaire controlatéral permet d'éviter la bascule prothétique. Le cas 6 (2 implants pour stabiliser une prothèse adjointe totale) peut être discuté : en effet, il existe un axe de rotation autour des deux implants. L'anatomie du greffon n'a pas permis de positionner plus d'implants. Pour les cas 7 et 10, l'anatomie buccale n'a pas permis de restaurer l'ensemble de l'arcade dentaire ; 2 implants ont donc été suffisants pour stabiliser la prothèse.

Cette série limitée constitue une étude préliminaire sur la mise en place d'implants dentaires dans des greffons de fibula microanastomosés, utilisés pour reconstruire la mandibule après une exérèse carcinologique, le plus souvent associée à une radiothérapie. Cette étude se poursuit et les résultats immédiats pour les nouveaux cas implantés sont encourageants. fibula, iliac crest and scapula of cadavers: preliminary report. Clin Oral Impl Res 1997 ; 8 : 286-9.

5 - Sclaroff A, Haughey B, Gay WD, Paniello R. Immediate mandibular reconstruction and placement of dental implants at the time of ablative surgery. Oral Surg Oral Med Oral Pathol $1994 ; 78$ : 711-7.

6 - Aldegheri A, Beloni D, Blanc JL, Kaplanski P, Legre L, ZANARET M. La réhabilitation dentaire par fixtures ostéointégrables : traitement des cancers oro-maxillo-faciaux. Rev Stomatol Chir Maxillofac 1996 ; 97 : 108-16.

7 - Serra JM, Paloma V, Mesa F, Ballesteros A. The vascularized fibula graft in mandibular reconstruction. J Oral Maxillofac Surg $1991 ; 49$ : 244-50. 
8 - Reychler H, Ortabe Jl, Pecheur A, Brogniez V. Mandibular reconstruction with a free vascularized fibula flap and osseointegrated implant: a report of four cases. J Oral Maxillofac Surg 1996 ; 54 : 1464-9.

9 - NaKAl H, NIIMI A, Ueda M. Histologic evaluation of clinical successful osseointegrated implants retrieved from irradiated bone: a report of 2 patients. Int $\mathrm{J}$ Oral Maxillofac Impl 1999 ; 14 : 442-6.

10 - SUMI Y, HASEGAWA T, MIYAISHI O, UEDA M. Interface analysis of titanium implants in a human vascularized fibula bone graft. J Oral Maxillofac Surg 2001 ; 59 : 213-6.

11 - POSNICK JC, WELLS MD, ZUCKER RM. Use of the free fibular flap in the immediate reconstruction of pediatric mandibular tumors: report of cases. J Oral Maxillofac Surg $1993 ; 51: 189-96$.

12 - Chiapasco M, Abati S, Ramundo G, Rossi A, Romeo E, VoGEL G. Behaviour of implants in bone grafts or free flaps after tumor resection. Clin Oral Impl Res 2000 ; $11: 66-75$.

13 - Matsuura M, Ohno K, Michi K, Egawa K, Takiguchi R. Clinicoanatomic examination of the fibula: anatomic basis for dental implant placement. Int J Oral Maxillofac Impl $1999 ; 14: 879-84$.

14 - Laure B, Van Hove A, Aboumoussa J, Gendre C, GogA D. Le lambeau libre de péroné. Anatomie. Technique de prélèvement. Rev Stomatol Chir Maxillofac $2000 ; 101: 147-53$

15 - GuerRA MFM, GIAS LN, ROdRIGUEZ FJ, CAMPO FJR, GONZALEZ FJD. Vascularized free fibular flap for mandibular reconstruction: a report of 26 cases. J Oral Maxillofac Surg 2001 : 140-4.

16 - HuRYN J, ZLOTOLOW IM, PIRO JD, LENCHEWSKI E. Osseointegrated implants in microvascular fibula free flap reconstructed mandible. J Prosth Dent 1993 ; 70 : 443-4.

17 - SCHWEIGER JW. Titanium implants in irradiated dog mandibles. J Prosth Dent $1989 ; 62$ : 201-5.

18 - Aisikanen P, Klemetti E, Kotilainen R, Vuillemin T, SUTtER F, VoIPIO HM, KULLAA A. Osteointegration of dental implants in bone irradiated with 40,50 or 60 Gy doses. Clin Oral Impl Res 1998 ; 9 : 20-5.
19 - Barber HD, Seckinger RJ, Hayden RE, Weinstein GS. Evaluation of osseointegration of endosseous implants in radiated, vascularized fibula flaps to the mandible. J Oral Maxillofac Surg 1995 ; 53 : 640-4.

20 - Brogniez V, Lejuste P, Pecheur A, Reychler H. Dental prosthetic reconstruction of osseointegrated implants placed in irradiated bone. Int J Oral Maxillofac Impl $1998 ; 13: 506-12$.

21 - CHEUNG LK, LEUNG AC. Dental implants in reconstructed jaws : implant longevity and peri-implant tissue outcomes. J Oral Maxillofac Surg 2003 ; 61 : 1263-74.

22 - ECKert SE, Desjardins RP, Keller EE, Tolman DE. Endosseous implants in an irradiated tissue bed. J Prosth Dent 1996 ; 76 : 45-9.

23 - ESSER E, WAGNER W. Dental implants following radical oral cancer surgery and adjuvant radiotherapy. JOMI $1997 ; 12: 552-61$.

24 - WeIscher T, Mohr C. Ten-year experience in oral implant rehabilitation of cancer patients: treatment concept and proposed criteria for success. Int J Oral Maxillofac Impl $1999 ; 14: 521-8$.

25 - Oechilin CK, Zimmermann AP, Grätzk W, Sailer HF. Histologic evidence of osseointegration in the irradiated and reconstructed mandible: a case report. Int J Oral Maxillofac Impl $1999 ; 14$ : 113-7.

26 - GRANStRÖM G, JacoBsson M, TJELLström A. Titanium implants in irradiated tissues: benefit from hyperbaric oxygen. Int J Oral Maxillofac Impl $1992 ; 7: 15-5$.

27 - TAYLOR TD, WORTHINGTON P. Osseointegrated implant rehabilitation of the previously irradiated mandible: results of a limited trial at 3 to 7 years. J Prosth Dent $1993 ; 69: 60-9$.

28 - Foster JH. Hyperbaric oxygen therapy: contraindications and complications. J Oral Maxillofac Surg 1992 ; $50: 1081-6$.

\section{médecine buccale chirurgie buccale \\ VOL. $11, \mathrm{~N}^{\circ} 4$ 2005}

page 221 